
\title{
25 Research Square \\ Is increased dose of triamcinolone for lumbar medial branch block associated with superior efficacy?
}

\section{Sukhee Park}

International St. Mary's Hospital, Catholic Kwandong University School of Medicine

\section{Su II Choi}

International St. Mary's Hospital, Catholic Kwandong University School of Medicine

Young Uk Kim ( $\sim$ uk201@hanmail.net)

International St. Mary's Hospital, Catholic Kwandong University School of Medicine

\section{Research Article}

Keywords: facet joint syndrome, medial branch nerve block, steroid, triamcinolone

Posted Date: February 23rd, 2022

DOI: https://doi.org/10.21203/rs.3.rs-1258870/v1

License: (c) (i) This work is licensed under a Creative Commons Attribution 4.0 International License.

Read Full License 


\section{Abstract}

Background: Although the beneficial effects of steroids for lumbar medial branch block (LMBB) have been demonstrated, there are no guidelines for selecting an appropriate steroid dose for LMBB. The study objective is to retrospectively compare the analgesic effect of different doses of triamcinolone in combination with local anesthetics to determine the lowest possible dose of triamcinolone for lumbar medial branch block in lumbar facet joint pain.

Methods: A total of 223 patients with clinical features of lumbar facet joint syndrome who underwent LMBB were retrospectively divided into 3 groups according to the triamcinolone doses, (1) 10-mg triamcinolone (T10 group, $n=121$ ); (2) 20-mg triamcinolone (T20 group, $n=87$ ); (3) 40-mg triamcinolone (T40 group, $n=15$ ). The outcome measurements were the numeric rating scale (NRS) pain scores, the 7point Likert satisfaction scale, and the proportion of responder (reduction in NRS pain score $>50 \%$ or 7 point Likert scale of $\geq 6$ ) at $2^{\text {nd }}$ visit after lumbar medial branch block.

Results: The change in NRS pain score at $2^{\text {nd }}$ visit $(25.6 \pm 14.0$ days) after LMBB showed no statistical difference between the three groups. The 7-point Likert scale at 2nd visit after the LMBB also did not show significant difference between the three groups. The proportion of responder was comparable among groups.

Conclusions: The increased dose of triamcinolone for LMBB did not show superior analgesic efficacy.

\section{Introduction}

The lumbar facet joint syndrome (LFJS) accounts for 15 to 40 percent of chronic back pain and is implicated as a major source of low back pain (LBP) [1-3]. While controversy remained regarding the proper management of LFJS, several studies have demonstrated the effectiveness of lumbar medial branch nerve blocks (LMBB) for LFJS [4-7].

The lumbar medial branch nerves supply the sensory innervation of the lumbar facet joints. LMBB with steroids and anesthetics may provide analgesic effect by the release of trapped nerve or suppression of ectopic discharges from inflamed medial branches, as well as reversal of central sensitization [8]. Although the beneficial effects of steroids for LMBB have been proved previous research, to our knowledge, there is no direct evidence to guide glucocorticoid dose selection, which mainly depends on physician's experience. Triamcinolone (TA) is a glucocorticoid used to treat certain skin diseases, rheumatic disorders and facet joint pain syndrome [9]. TA is a glucocorticoid that is about five times as potent as cortisol but has very little mineralocorticoid effects. Depending on the route of administration and the treated condition, the onset of action can be from two hours up to one or two days after application; and the drug can act much longer than its elimination half-life would suggest. However, Side effects of TA long-term treatment may include sinusitis, osteoporosis, reduced muscle mass, metabolic syndrome-like symptom, and life-threatening side effects such as adrenal suppression, immunosuppression, and steroid-induced hyperglycemia [4, 10-12]. Now we also should consider critical 
questions regarding the potential effects of interventional treatments utilizing steroids on COVID-19 vaccines safety and efficacy [13]. Therefore, it is important to find an appropriate steroid dose that minimizes side effects while maintaining analgesic effects.

This study objective is to compare the therapeutic effect of different doses of TA in combination with local anesthetics to investigate whether the dose of TA for LMBB is related to efficacy in lumbar facet joint originating pain.

\section{Methods}

This study was a retrospective analysis of electrical medical records. Ethical approval was provided by the Institutional Review Board (IRB) of Catholic Kwandong University International St. Mary's Hospital (Approval number: IS20RISI0080). The requirement for written informed consent was waived due to the retrospective nature of the study. This study is reported in accordance with STROBE guidelines. The methodology in this study followed the Strengthening the Reporting of Observational Studies in Epidemiology (STROBE) Statement.

\section{Patient population}

Adult patients who underwent LMBB between March 2015 and February 2017 in single hospital were enrolled. LMBB was performed when there was no clinical evidence of nerve root irritation sign originating from an intervertebral disc with confirmatory magnetic resonance imaging studies and when conservative treatments were ineffective and LBP of facet joint origin was clinically suspected (e.g., paraspinal tenderness and increasing pain on lumbar extension). We only included the patients with LBP for at least 3 months who underwent at least 4 LMBB and who did not receive other epidural steroid injections for 4 weeks before and after LMBB. Despite taking oral opioid analgesics, patients with refractory LBP with a number rating scale (NRS) of 7 or more were not included. The Patients were classified into those who used 10-mg TA (T10 group), 20-mg TA (T20 group), and 40-mg TA (T40 group).

\section{Procedures}

Patients were positioned prone on a radiolucent fluoroscopy table. Non-invasive blood pressure monitoring and a pulse oximeter were placed. The lumbar spine was prepared with povidone and draped in a sterile manner. Fluoroscopy was used to identify the appropriate lumbar levels. The skin and subcutaneous tissues superficial to each medial branch target were anesthetized with $0.2 \mathrm{~mL}$ of $1 \%$ lidocaine. A 23-gauge spinal was placed at the junction of the transverse process and the superior articular process in an ipsilateral oblique fluoroscopic view. Appropriate positioning was confirmed in anterior-posterior and lateral fluoroscopic views. Approximately $0.5 \mathrm{~mL}$ of 10,20 or $40-\mathrm{mg}$ of triamcinolone mixed with $1 \%$ lidocaine was injected per each LMB.

\section{Data acquisition}


Data was obtained from electrical medical charts. We collected demographic data including age, gender, and comorbidities; pre-procedural data including NRS pain scores $(0=$ no pain and $10=$ most severe pain imaginable) and the patient satisfaction was assessed using a 7-point Likert scale ( $1=$ extremely dissatisfied, 2 = dissatisfied, 3 = somewhat dissatisfied, 4 = Undecided, 5 = somewhat satisfied, 6 = satisfied, and $7=$ extremely satisfied). Procedural information including injected steroid dose and post procedural information including NRS pain scores and 7-point Likert scale at 2nd visit after LMBB were collected. We defined more than a 50\% reduction of the NRS score or 7-point Likert scale of 6 or higher by the second visit as a responder.

\section{Statistical analysis}

Continuous variables were summarized as mean (standard deviation), and categorical variables were presented as frequency (\%). Continuous variables were analyzed using One-way analysis of variance or Kruskal-Wallis test combined with Tukey test using ranks for post hoc testing. Categorical variables were analyzed using chi-square test or Fisher's exact text. All reported $P$-values were two-sided, and $P$-values < 0.05 was considered statistically significant. Analyses were performed using SPSS 25.0 (IBM Corp., Chicago, IL).

\section{Results}

Based on inclusion criteria, a total of 223 patients were finally analyzed. T10 group had 121, T20 group had 87, and T40 group had 15 patients. There were no intergroup differences with respect to gender, age, hypertension, diabetes mellitus and previous lumbar spinal surgery history (Table 1).

Table 1

Comparison of the characteristics of each group.

\begin{tabular}{|lllll|}
\hline & $\begin{array}{l}\text { T10 Group } \\
(\mathbf{n = 1 2 1 )}\end{array}$ & $\begin{array}{l}\text { T20 Group } \\
(\mathbf{n}=\mathbf{8 7})\end{array}$ & $\begin{array}{l}\text { T40 Group } \\
(\mathbf{n = 1 5 )}\end{array}$ & p-value \\
\hline Male (\%) & $81(66.9)$ & $53(60.9)$ & $11(73.3)$ & 0.151 \\
\hline Age (years) & $56.7 \pm 17.0$ & $53.9 \pm 16.0$ & $47.7 \pm 15.6$ & 0.103 \\
\hline Hypertension (\%) & $31(25.8)$ & $16(18.8)$ & $2(13.3)$ & 0.340 \\
\hline Diabetes mellitus (\%) & $18(15.0)$ & $10(11.8)$ & $1(6.7)$ & 0.591 \\
\hline Previous lumbar spinal surgery (\%) & $14(15.7)$ & $15(17.2)$ & 0 & 0.146 \\
\hline Data are presented as mean \pm standard deviation or frequency (percent). & \\
\hline
\end{tabular}

The mean interval from LMBB to the follow up evaluation was $25.6 \pm 14.0$ days, and it did not show significant difference between the three groups (T10, $25.0 \pm 15.1$ days; T20, $25.8 \pm 14.7$ days; and T40, $27.3 \pm 10.4$ day; $P=0.784)$. The mean pre-procedural NRS pain score was 4.7 and it was not statistically different between the three groups (T10, $4.8 \pm 1.2 ; \mathrm{T} 20,4.7 \pm 1.0$; and T40, $4.8 \pm 1.0 ; P=0.861)$. The 
change in NRS pain score after LMBB significantly decreased in among groups (Fig. 1). However, there was no significant difference among groups at 2 nd visit after the LMBB $(T 10,2.8 \pm 1.0 ; T 20,3.0 \pm 1.1$; and T40, $2.7 \pm 1.2 ; P=0.463)$. Similarly, the 7-point Likert scale at 2 nd visit after the LMBB did not show significant difference between the three groups $(P=0.482$; Fig. 2$)$. The post-hoc analysis showed no intergroup differences in both NRS pain score and the 7-point Likert scale at 2nd visit.

The proportion of responder at 2nd visit was comparable among groups (Table 2). The proportion of patients achieving at least a $50 \%$ reduction in NRS pain score at 2 nd visit after the LMBB did not show significance $(\mathrm{T} 10,39.7 \% ; \mathrm{T} 20,29.9 \%$; and $\mathrm{T} 40,33.3 \% ; P=0.342)$. The proportion of patients reporting a 7point Likert satisfaction scale $\geq 6$ increased with increasing dose of TA, but there was no statistically significant difference between the 3 dosing regimens (T10,38.0\%; T20, 47.1\%; and T40, 53.3\%; $P=$ 0.290). There were no adverse central neurologic outcomes and/or cardiopulmonary compromise in the recovery area.

Table 2

Patients experiencing at least a post-procedural $50 \%$ reduction in NRS pain score or 7-point Likert satisfaction scale of $\geq 6$.

\begin{tabular}{|lllll|}
\hline & $\begin{array}{l}\text { T10 Group } \\
(\mathbf{n = 1 2 1 )}\end{array}$ & $\begin{array}{l}\text { T20 Group } \\
(\mathbf{n = 8 7})\end{array}$ & $\begin{array}{l}\text { T40 Group } \\
(\mathbf{n = 1 5 )}\end{array}$ & $P$-value \\
\hline Responder & $66(55.5)$ & $47(54.0)$ & $9(60.0)$ & 0.909 \\
\hline $50 \%$ reduction in NRS score (\%) & $48(39.7)$ & $26(29.9)$ & $5(33.3)$ & 0.342 \\
\hline 7-point Likert satisfaction scale of $\geq 6$ & $46(38.0)$ & $41(47.1)$ & $8(53.3)$ & 0.290 \\
\hline $\begin{array}{l}\text { Data are presented as frequency (percent). Likert scale where 1 = extremely dissatisfied, 2 = } \\
\text { dissatisfied, 3 = somewhat dissatisfied, 4 = Undecided, 5 = somewhat satisfied, 6 = satisfied, and 7 = } \\
\text { extremely satisfied. }\end{array}$ \\
\hline
\end{tabular}

\section{Discussion}

No studies to date have compared the efficacy of different dose of TA during LMBB in LFJS. Our results showed that there was no change in the pain score even when the steroid dose was increased. The patient's satisfaction after LMBB increased with increasing dose of TA, but there was no statistical difference, suggesting that there might not be necessary to use high dose of steroids for LMBB considering risk-benefit ratio.

The facet joint capsules are rich in both the type c- and A-delta nerve fibers endings and the pain receptors are easily sensitized [14]. Neurophysiologic studies have shown that facet joint capsules also contain low-threshold mechanically sensitive nociceptors [14, 15]. Inflammation of facet joint leads to decreased thresholds of nerve endings in facet joint capsules as well as elevated baseline discharge rates. 
LFJS is associated with a good response to LMBB. There are several explanations for the effective pain relief observed after LMBB in patients with LFJS. Park et al. have insisted that disc degeneration correlated with facet joint force [16]. As the facet joint and intervertebral disc is a three-joint complex, degenerative change of the disc can affect facet joint loading. Like the effect of another block to a loaded structure, LMBB may be effective for increasing facet loading accompanied with disc degeneration $[6,7]$. Also, it is possible that injectate from the LMBB procedure spread to adjacent structures, such as intervertebral discs or back muscles, which may have been causing axial discomfort, and this may have affected the response to LMBB [16].

The steroids exert effectiveness by anti-inflammatory, immunosuppressive, anti-erosion, and inhibition of neurotransmitter within c-nerve fibers $[2,14,17,18]$. Especially, TA is a glucocorticoid that is about five times as potent as cortisol, but has very little mineralocorticoid effects [12]. Additionally, the local anesthetics play an important role in reducing central sensitization by inhibiting the emission of harmful signals from nerve ending and conduction to sympathetic nerve fibers $[5,18]$. These two similar but different mechanisms could explain the effect of local anesthetic and TA combination in LMBB.

However, the side effects of steroids diverse from self-limited minor side effects such as facial flushing and headaches to potentially more serious adverse events including steroid-induced hyperglycemia which has been found to be dose dependent [18]. Other steroid-related side effects have also been reported. Multiple studies have found that suppression of the hypothalamic-pituitary axis [19]. To best mitigate these adverse events caused by steroids, the physician should limit cumulative exposure to steroids and avoid unnecessarily high doses and aware of a minimally effective dose [20]. Patients at risk of these complications, including patients with diabetes or adrenal insufficiency, should also be identified as being at increased risk [1]. In addition, we now faced questions regarding the potential effects of steroids on COVID-19 vaccines. Although there is no evidence to date that interventional pain management with steroids affects the efficacy of the COVID-19 vaccine or increases the risk of side effects from the vaccine [13], unnecessarily high doses should be avoided because steroid side effects increase with increasing dose [18].

This study has several limitations. First, as a retrospective study, we could not exclude the possibility of bias from unobserved (unmeasured or unmeasurable) variables. For instance, the variables such as dosedependent side effects of steroid, physical therapy, and rehabilitation were not reliably charted and could not be analyzed. Second, addition of a functional metric, such as the Oswestry low back pain questionnaire, could potentially clarify the results. This study is meant to be a reference for the future design of randomized controlled trials to conclusively elucidate the relationship between TA dose for LMBB and quantification of pain relief. Third, we included the small number of patients and the lack of a control group of patients not on steroids during therapy. Forth, we analyzed the effect of TA at short-term follow-up and compared the results of performing LMBB only once, which has a probability of false positive rates. Future studies should closely examine the effect of TA on long term period. Finally, only an association but not causation can be determined. Large prospective studies are indicated to confirm the optimal dose of TA for LMBB. 


\section{Conclusions}

In conclusion, increasing the dose of TA used for LMBB in LFJS did not show superior analgesic effect. A standardized TA dose protocol in LMBB will be necessary.

\section{Abbreviations}

LMBB

lumbar medial branch block

LFJS

lumbar facet joint syndrome

LBP

low back pain

TA

triamcinolone

NRS

numeric rating scale

\section{Declarations}

\section{Ethics approval and consent to participate}

This study was a retrospective analysis of electrical medical records. Ethical approval was provided by the Institutional Review Board (IRB) of Catholic Kwandong University International St. Mary's Hospital (Approval number: IS20RISI0080). The requirement for written informed consent was waived due to the retrospective nature of the study. The methodology in this study followed the Strengthening the Reporting of Observational Studies in Epidemiology (STROBE) Statement.

\section{Consent for publication}

Not applicable.

\section{Availability of data and materials}

Restrictions apply to the availability of these data, as it is the International St. Mary's Hospital policy not to make outcome data publicly available. However, the datasets analysed during the current study are available from the corresponding author on reasonable request and with the permission of International St. Mary's Hospital administration.

\section{Competing interests}

The authors of this study declare that there are no conflicts of interest. 


\section{Funding}

The authors of this study declare that they have not received financial support from any source.

\section{Authors' contributions}

Park S and Kim YU have participated to design the study and write and edit the final version of the manuscript. Park S, Choi SI, and Kim YU have participated to collect and analyse the data. All authors read and approved the final version of the manuscript.

\section{References}

1. Bellomo RG, Paolucci T, Giannandrea N, Pezzi L, Saggini R: Ozone Therapy and Aquatic Rehabilitation Exercises to Overcome the Lumbar Pain Caused by Facet Joint Syndrome - Case Report. Int Med Case Rep J 2020, 13:171-176.

2. Piraccini E, Calli M, Corso RM, Byrne H, Maitan S: Abdominal and pelvic pain: an uncommon sign in lumbar facet joint syndrome. Minerva Anestesiol 2017, 83(1):104-105.

3. Sowa G: Facet-mediated pain. Dis Mon 2005, 51(1):18-33.

4. Mohan A, Tijmes S, Cohen J, Sherman EM, Eckardt P: latrogenic Cushing syndrome following lumbar medial branch block in a patient with HIV on ritonavir and darunavir. Pain Manag 2021.

5. Finlayson RJ, Curatolo M: Consensus practice guidelines on interventions for lumbar facet joint pain: finding a path through troubled waters. Reg Anesth Pain Med 2020, 45(6):397-398.

6. Manchikanti L, Singh V, Falco FJ, Cash KA, Pampati V: Evaluation of lumbar facet joint nerve blocks in managing chronic low back pain: a randomized, double-blind, controlled trial with a 2-year followup. Int J Med Sci 2010, 7(3):124-135.

7. Manchikanti L, Singh V, Falco FJ, Cash KA, Pampati V: Lumbar facet joint nerve blocks in managing chronic facet joint pain: one-year follow-up of a randomized, double-blind controlled trial: Clinical Trial NCT00355914. Pain physician 2008, 11(2):121-132.

8. Cavanaugh JM, Lu Y, Chen C, Kallakuri S: Pain generation in lumbar and cervical facet joints. J Bone Joint Surg Am 2006, 88 Suppl 2:63-67.

9. Ganapathy PS, Lowder CY, Arepalli S, Baynes K, Li M, Bena J, Srivastava SK: Treatment Duration and Side Effect Profile of Long-Term Use of Intravitreal Preservative-Free Triamcinolone Acetonide in Uveitis. Am J Ophthalmol 2018, 194:63-71.

10. Schneider BJ, Maybin S, Sturos E: Safety and Complications of Cervical Epidural Steroid Injections. Phys Med Rehabil Clin N Am 2018, 29(1):155-169.

11. Friedly JL, Comstock BA, Heagerty PJ, Bauer Z, Rothman MS, Suri P, Hansen R, Avins AL, Nedeljkovic SS, Nerenz DR et al: Systemic effects of epidural steroid injections for spinal stenosis. Pain 2018, 159(5):876-883. 
12. Suarez-Figueroa M, Contreras I, Noval S: [Side-effects of triamcinolone in young patients]. Arch Soc Esp Oftalmol 2006, 81(7):405-407.

13. Chakravarthy K, Strand N, Frosch A, Sayed D, Narra LR, Chaturvedi R, Grewal PK, Pope J, Schatman ME, Deer T: Recommendations and Guidance for Steroid Injection Therapy and COVID-19 Vaccine Administration from the American Society of Pain and Neuroscience (ASPN). Journal of pain research 2021, 14:623-629.

14. Cohen SP, Bhaskar A, Bhatia A, Buvanendran A, Deer T, Garg S, Hooten WM, Hurley RW, Kennedy DJ, McLean $B C$ et al: Consensus practice guidelines on interventions for lumbar facet joint pain from a multispecialty, international working group. Regional anesthesia and pain medicine 2020, 45(6):424467.

15. Cohen SP, Doshi TL, Constantinescu OC, Zhao Z, Kurihara C, Larkin TM, Griffith SR, Jacobs MB, Kroski WJ, Dawson TC et al: Effectiveness of Lumbar Facet Joint Blocks and Predictive Value before Radiofrequency Denervation: The Facet Treatment Study (FACTS), a Randomized, Controlled Clinical Trial. Anesthesiology 2018, 129(3):517-535.

16. Park JY, Kim DH, Seo DK, Yoon SH, Lee G, Lee S, Park CH, Sim SE, Suh JH: Predictors of Response to a Medial Branch Block: MRI Analysis of the Lumbar Spine. J Clin Med 2019, 8(4).

17. Lee HM, Weinstein JN, Meller ST, Hayashi N, Spratt KF, Gebhart GF: The role of steroids and their effects on phospholipase A2. An animal model of radiculopathy. Spine (Phila Pa 1976) 1998, 23(11):1191-1196.

18. Johansson A, Hao J, Sjölund B: Local corticosteroid application blocks transmission in normal nociceptive C-fibres. Acta anaesthesiologica Scandinavica 1990, 34(5):335-338.

19. Kim WH, Sim WS, Shin BS, Lee CJ, Jin HS, Lee JY, Roe HJ, Kim CS, Lee SM: Effects of two different doses of epidural steroid on blood glucose levels and pain control in patients with diabetes mellitus. Pain physician 2013, 16(6):557-568.

20. Younes M, Neffati F, Touzi M, Hassen-Zrour S, Fendri Y, Béjia I, Ben Amor A, Bergaoui N, Najjar MF: Systemic effects of epidural and intra-articular glucocorticoid injections in diabetic and non-diabetic patients. Joint Bone Spine 2007, 74(5):472-476.

\section{Figures}




\section{NRS pain scores}

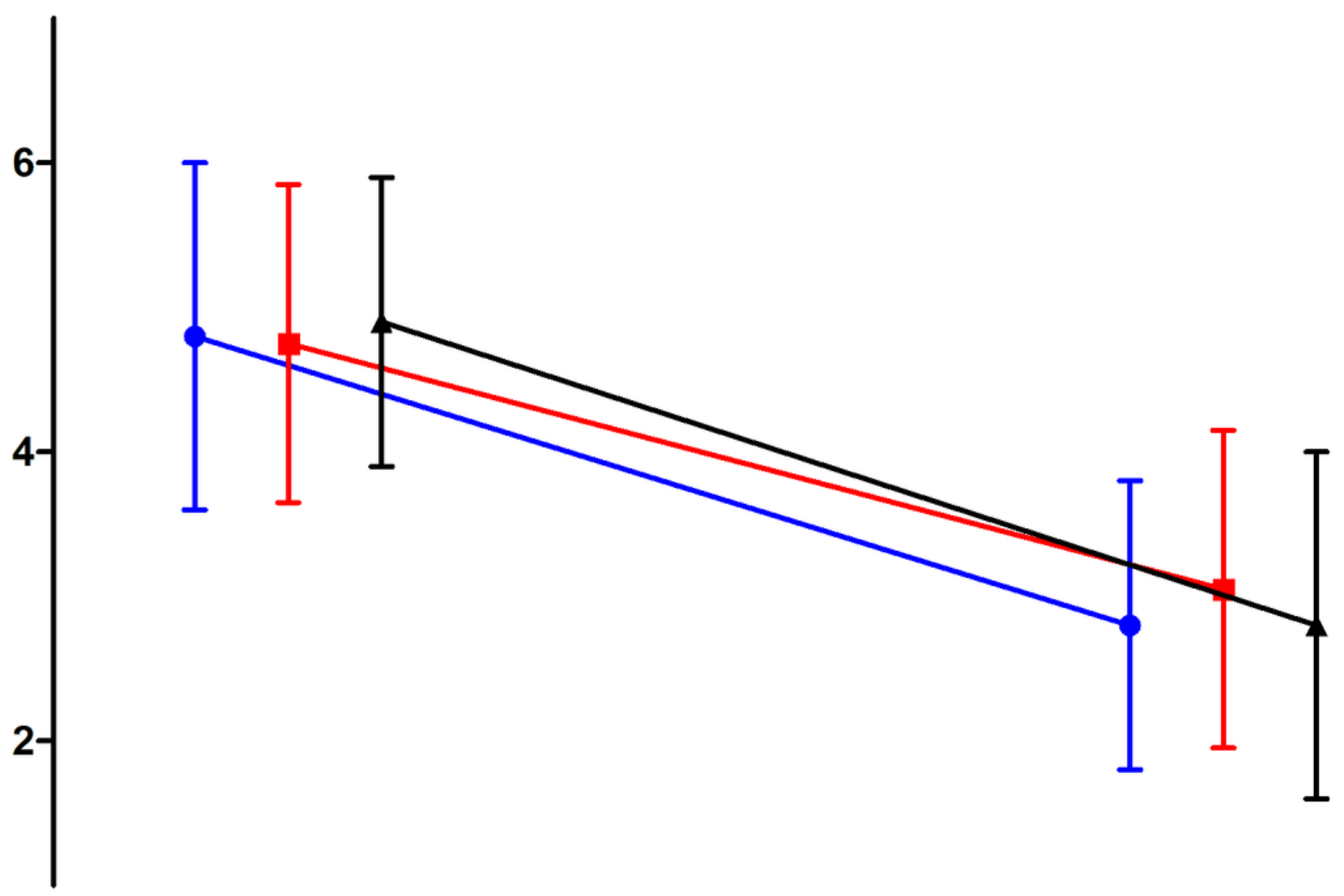

Baseline

$\rightarrow \quad$ T10 Group $2^{\text {nd }}$ vist after LMBB

- T30 Group

Figure 1

The scores for severity of back pain as measured on a numeric rating scale (NRS) pain score, after lumbar median branch block (LMBB) in patients with degenerative facet joint syndrome. 


\section{Percentage}

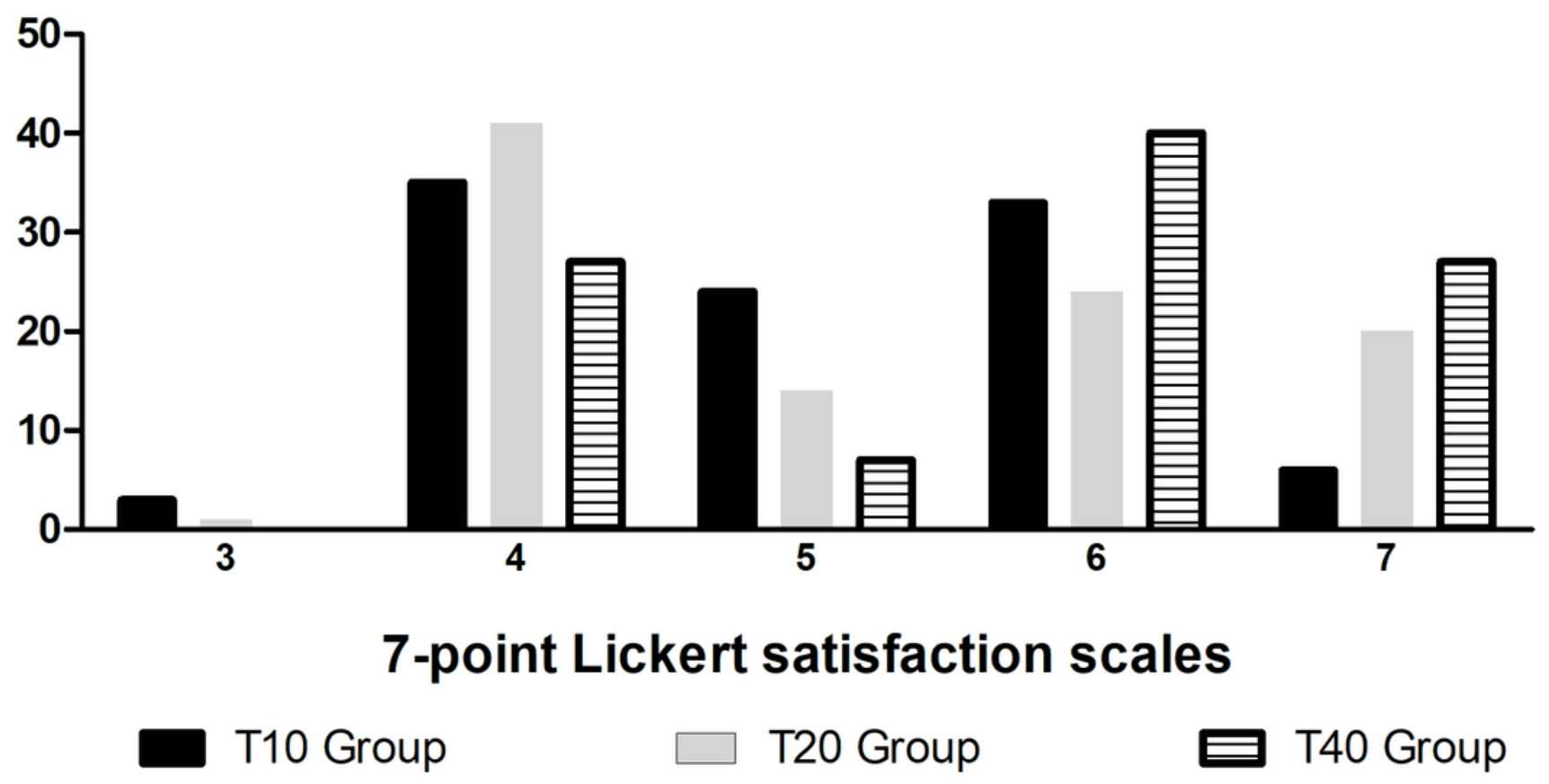

Figure 2

The 7-point Likert satisfaction scales after lumbar median branch block in patients with degenerative facet joint syndrome. 